\title{
The Solar Photospheric Nitrogen Abundance: Determination with 3D and 1D Model Atmospheres
}

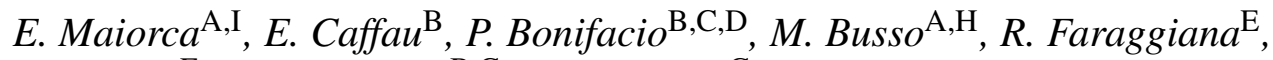 \\ M. Steffen ${ }^{\mathrm{F}}$, H.-G. Ludwig ${ }^{\mathrm{B}, \mathrm{C}}$, and I. Kamp ${ }^{\mathrm{G}}$ \\ A Dipartimento di Fisica, Università degli studi di Perugia, via Pascoli, Perugia, I-06123, Italy

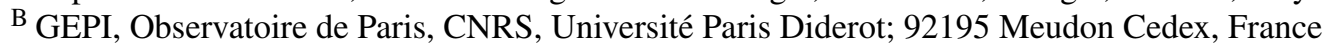 \\ ${ }^{\mathrm{C}}$ CIFIST Marie Curie Excellence Team \\ ${ }^{D}$ Istituto Nazionale di Astrofisica, Osservatorio Astronomico di Trieste, Via Tiepolo 11, \\ I-34143 Trieste, Italy \\ ${ }^{\text {E }}$ Istituto Nazionale di Fisica Nucleare, section of Perugia, via Pascoli, Perugia, I-06123, Italy \\ F Dipartimento di Astronomia, Università degli Studi di Trieste, via G.B. Tiepolo 11, \\ 34143 Trieste, Italy \\ G Astrophysikalisches Institut Potsdam, An der Sternwarte 16, D-14482 Potsdam, Germany \\ ${ }^{\mathrm{H}}$ Kapteyn Astronomical Institute, Postbus 800, 9700 AV Groningen \\ ${ }^{\text {I }}$ Corresponding author. Email: emaiorca@ gmail.com
}

Received 2008 November 26, accepted 2009 January 23

\begin{abstract}
We present a new determination of the solar nitrogen abundance making use of 3D hydrodynamical modelling of the solar photosphere, which is more physically motivated than traditional static 1D models. We selected suitable atomic spectral lines, relying on equivalent width measurements already existing in the literature. For atmospheric modelling we used the $\mathrm{CO}^{5} \mathrm{BOLD} 3 \mathrm{D}$ radiation hydrodynamics code. We investigated the influence of both deviations from local thermodynamic equilibrium (non-LTE effects) and photospheric inhomogeneities (granulation effects) on the resulting abundance. We also compared several atlases of solar flux and centre-disc intensity presently available. As a result of our analysis, the photospheric solar nitrogen abundance is $A(\mathrm{~N})=7.86 \pm 0.12$.
\end{abstract}

Keywords: hydrodynamics - line: formation — radiative transfer — Sun: abundances — Sun: granulation - Sun: atmosphere

\section{Introduction}

Images of the Sun reveal a very complex surface structure, which is referred to as granulation, and may be understood as the signature of the convective motions in the photosphere. Traditional static 1D model photospheres ignore all this complex phenomenology. In the last ten years hydrodynamical simulations of stellar photospheres have considerably improved and are now at the stage that they can be compared realistically against observations. This class of models (here and after referred to as '3D models') is physically better motivated, although computationally considerably more demanding, than traditional static 1D models. For the Sun, the comparison of present 3D models with observations shows encouraging agreement (e.g. Figure 1). The application of 3D models for abundance work is a largely unexplored territory, but promising work is in progress not only for the Sun but also for other solar-type stars. We began concentrating on chemical abundance determinations based on the analysis of high resolution spectra and the use of 3D models. The solar abundances clearly occupy a prominent place in this project, since we are able to obtain spectra of very high resolution and $\mathrm{S} / \mathrm{N}$ ratio for the Sun. The pioneering works in this field by Allende Prieto, Lambert \& Asplund (2001) and Asplund et al. (2004) have led to a substantial downward revision of the solar metallicity, which implies an awkward tension with the helioseismic measurements (see Basu \& Antia 2008, and references therein). It is thus not unsurprising that we start our investigation by a reassessment of the abundances of the main contributors to the solar metallicity, $Z$, i.e. oxygen, nitrogen and carbon. The present contribution reports on a redetermination of the solar nitrogen abundance based on atomic lines.

In Asplund, Grevesse \& Sauval (2005), among other elements, the solar abundance of nitrogen was also considered, based on a 3D model. Unfortunately not much information was given as to which lines are used, oscillator strengths and other details of the analysis. Both molecular and atomic lines were considered, giving an abundance which is $7.73 \pm 0.05$ and $7.85 \pm 0.08$, respectively (including NLTE corrections). 

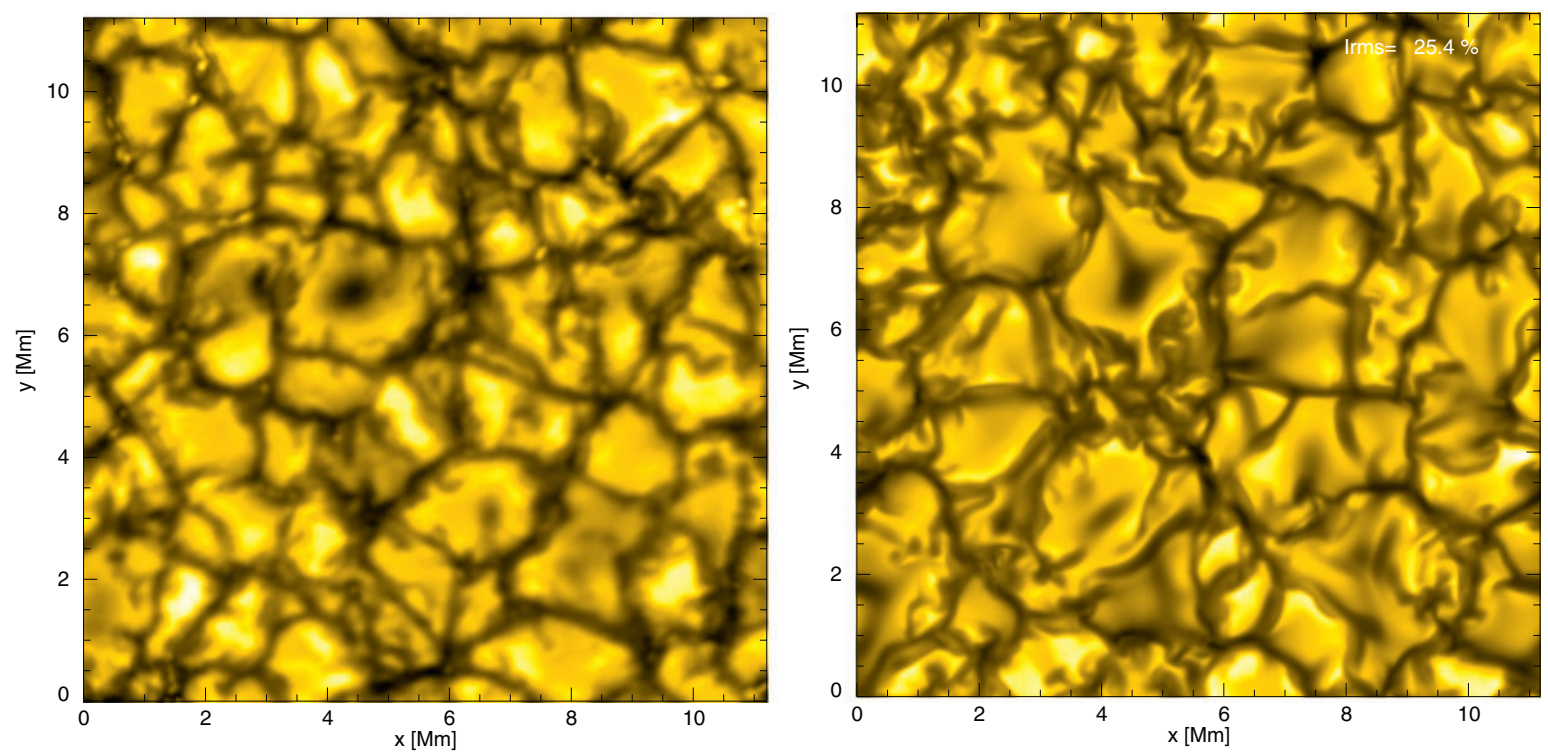

Figure 1 Left: Quiet solar granulation as observed with the 1-m Swedish Solar Telescope (courtesy Mats Carlsson 2004). Right: High-resolution $\mathrm{CO}^{5}$ BOLD simulation of solar surface convection. Both images show the emergent continuum intensity (using identical scaling) at $\lambda 4364 \AA$ in a field measuring $15^{\prime \prime} \times 15^{\prime \prime}(11 \times 11 \mathrm{Mm})$.

\section{Models and Line Formation Codes}

Our analysis is mainly based on a 3D model computed with the CO ${ }^{5}$ BOLD code (Freytag, Steffen \& Dorch 2002; Freytag et al. 2003; Wedemeyer et al. 2004). Some basic information on the setup of this numerical simulation can be found in Caffau et al. (2008), who used the same solar model to determine the solar oxygen abundance. We just point out the basic differences between the new approach using 3D models and the old approach using 1D models: while 1D models describe a time-independent, hydrostatic atmosphere, a 3D model is the result of solving numerically the time-dependent hydrodynamic equations together with the equation of radiation transport. For any instant of time ('snapshot' in 3D-jargon) the 3D models give the physical quantities on a 3D mesh of points in the photosphere. This allows a more realistic description of the atmosphere (see Figure 1), since both vertical and horizontal fluctuations of physical quantities can be taken into account. Moreover, the (turbulent) velocity field in the stellar atmosphere is automatically obtained without the need to specify free parameters (like micro- and macroturbulence). We would like to point out that, although not requiring the free parameters needed in $1 \mathrm{D}$ computations to adjust the efficiency of the convective energy transport and the strength of the turbulent velocity field, 3D models are characterized by a set of numerical parameters, e.g. the numerical scheme used for solving the hydrodynamical equations, the spatial resolution of the numerical grid, the amount of artificial viscosity, the number of rays (angles) considered in computing the radiation field and many others. The hope is that the results become essentially independent of the choice of these parameters once the numerical resolution exceeds some critical threshold.

Besides the $\mathrm{CO}^{5}$ BOLD model, we considered also several 1D models for comparison. These include the semi-empirical Holweger-Müller model (Holweger 1967; Holweger \& Müller 1974, hereafter HM), a 1D model computed with the LHD code (see Caffau \& Ludwig 2007 for further details), an ATLAS solar model computed by F. Castelli ${ }^{1}$, and several 1D models with solar parameters computed by ourselves with Version 9 of the aTLas code (Kurucz 1993a, 2005b) in its LINUx version (Sbordone et al. 2004; Sbordone 2005), using the 'NEW' Opacity Distribution Functions (ODFs; Castelli \& Kurucz 2003) and, finally, a 1D model obtained by temporal and spatial averaging of the 3D model over surfaces of equal (Rosseland) optical depth, which we call $\langle 3 \mathrm{D}\rangle$ model.

The 3D spectrum synthesis computations are all performed with $\operatorname{LINFOR}_{3} \mathrm{D}^{2}$, which can also compute line formation using different kinds of 1D models as input. For comparison, in the case of 1D models we also used the WIDTH code for calculating equivalent widths (Kurucz 1993a, 2005b; Castelli 2005; Sbordone 2005) and the SYNTHE code in its LINUX version (Kurucz 1993b, 2005b; Sbordone et al. 2004; Sbordone 2005) for calculating synthetic spectra.

\section{Line Selection, Atomic and Observational Data}

In the literature one can find different choices for the $\log g f$ values (see Table 1). We decided in favour of the NIST data, first of all because, in this database both computed and measured values are critically examined; second because all elements ara available; and third, to be consistent with the other papers we produced on photospheric solar abundances.

\footnotetext{
${ }^{1}$ http://wwwuser.oats.inaf.it/castelli/sun/ apo0t5777g44377k1asp.dat.

2 http://www.aip.de/ mst/Linfor3D/linfor_3D_ manual.pdf.
} 
Table 1. Compilation of $\log g f$ values of permitted NI lines used by different authors

\begin{tabular}{|c|c|c|c|c|c|c|c|c|c|}
\hline \multirow{2}{*}{$\begin{array}{l}\lambda \\
(\mathrm{nm})\end{array}$} & \multirow[t]{2}{*}{ NIST } & \multicolumn{2}{|c|}{ Lambert 68} & \multirow[t]{2}{*}{ Lambert 78} & \multicolumn{2}{|c|}{ Biémont } & \multirow[t]{2}{*}{ Grevesse } & \multirow[t]{2}{*}{ Takeda } & \multirow[t]{2}{*}{ Rentzsch-Holm } \\
\hline & & $\mathrm{CA}$ & NBS & & $\mathrm{L}$ & V & & & \\
\hline 744.2 & $-0.385 \mathrm{~B}+$ & -0.33 & -0.45 & -0.33 & -0.387 & -0.463 & -0.411 & -0.386 & -0.573 \\
\hline 746.8 & $-0.190 \mathrm{~B}+$ & -0.16 & -0.27 & -0.16 & -0.171 & -0.248 & -0.208 & -0.171 & -0.397 \\
\hline 818.4 & $-0.286 \mathrm{~B}+$ & -0.23 & -0.42 & & & & & & \\
\hline 820.0 & $-1.001 \mathrm{~B}+$ & & & & & & -0.996 & -1.017 & -1.090 \\
\hline 821.6 & $+0.132 \mathrm{~B}+$ & +0.13 & -0.01 & +0.13 & +0.146 & +0.089 & -0.106 & +0.147 & +0.012 \\
\hline 822.3 & $-0.271 \mathrm{~B}+$ & & & & & & -0.288 & -0.267 & -0.390 \\
\hline 824.2 & $-0.256 \mathrm{~B}+$ & & & & & & -0.260 & -0.262 & -0.360 \\
\hline 859.4 & $-0.334 \mathrm{~B}$ & -0.32 & -0.38 & -0.32 & -0.320 & -0.332 & & & \\
\hline 862.9 & $+0.075 \mathrm{~B}$ & +0.08 & +0.03 & +0.08 & +0.090 & +0.078 & +0.082 & +0.090 & +0.069 \\
\hline 865.5 & $-0.627 \mathrm{~B}$ & -0.62 & -0.65 & -0.62 & -0.603 & -0.616 & -0.608 & -0.603 & -0.630 \\
\hline 868.3 & $+0.087 \mathrm{~B}+$ & +0.11 & -0.05 & +0.11 & +0.115 & +0.102 & +0.109 & +0.116 & -0.051 \\
\hline 870.3 & $-0.321 \mathrm{~B}+$ & -0.29 & -0.41 & -0.29 & & & & & \\
\hline 871.1 & $-0.234 \mathrm{~B}+$ & -0.18 & -0.34 & & & & & & \\
\hline 871.8 & $-0.336 \mathrm{~B}+$ & -0.26 & -0.43 & -0.26 & -0.338 & -0.347 & -0.344 & -0.337 & -0.419 \\
\hline 904.5 & $+0.439 \mathrm{~B}$ & & & & & & +0.430 & +0.429 & \\
\hline 939.2 & $+0.320 \mathrm{~B}$ & +0.31 & +0.24 & +0.31 & +0.328 & +0.378 & +0.354 & +0.328 & +0.316 \\
\hline 1010.5 & $+0.219 \mathrm{~B}+$ & & & & & & +0.220 & +0.234 & +0.200 \\
\hline 1010.8 & $+0.431 \mathrm{~B}+$ & +0.39 & +0.41 & +0.39 & +0.443 & +0.420 & +0.431 & +0.443 & +0.403 \\
\hline 1011.2 & $+0.607 \mathrm{~B}+$ & +0.58 & +0.60 & +0.58 & +0.622 & +0.600 & +0.611 & +0.623 & +0.588 \\
\hline 1011.4 & $+0.768 \mathrm{~B}+$ & +0.74 & +0.76 & +0.74 & +0.778 & +0.755 & +0.766 & +0.778 & +0.751 \\
\hline 1050.7 & $+0.094 \mathrm{~B}$ & & & & & & +0.249 & +0.249 & +0.250 \\
\hline 1052.0 & $+0.010 \mathrm{~B}$ & & & & & & -0.045 & +0.010 & -0.040 \\
\hline 1053.9 & $+0.503 \mathrm{~B}$ & +0.52 & +0.51 & & & & +0.529 & +0.525 & +0.530 \\
\hline 1075.7 & $-0.608 \mathrm{C}+$ & & & & & & -0.098 & -0.098 & -0.080 \\
\hline 1238.1 & $+0.247 \mathrm{C}+$ & & & & & & +0.284 & +0.175 & +0.320 \\
\hline 1246.1 & $+0.480 \mathrm{~B}$ & & & & & & +0.463 & +0.437 & +0.451 \\
\hline 1246.9 & $+0.629 \mathrm{~B}$ & & & & & & +0.622 & +0.622 & +0.610 \\
\hline
\end{tabular}

Notes. In 'Lambert 68' CA stands for 'theoretical Coulomb approximation', NBS (National Bureau of Standards) is the old NIST. In 'Biémont' L stands for 'length formalism', $\mathrm{V}$ for 'velocity formalism'.

Our chosen line list and oscillator strengths are given in Table 2.

\section{Results from Line-Profile Fitting Using Different Solar Atlases}

We also derived the nitrogen abundance from line profile fitting for few selected lines. We did this exercice for comparing the abundance derived from the four high resolution, high $\mathrm{S} / \mathrm{N}$, solar atlases available, the two centre-disc intensity atlases (the 'Delbouille' atlas, i.e. Delbouille, Roland \& Neven 1973 and Delbouille et al. 1981 and the 'Neckel' intensity atlas, Neckel \& Labs 1984) as well as the two solar-flux atlases (the 'Kurucz' solarflux atlas, Kurucz 2005a and the 'Neckel' solar-flux atlas, Neckel \& Labs 1984). From previous investigations of the observed solar spectra (see Caffau et al. 2008) we know that these atlases do not always agree. In Figure 2 we can see that the two centre-disc solar atlases are not in agreement for the line at $\lambda 821.6 \mathrm{~nm}$. The solar nitrogen abundance could be deduced from line profile fitting, but the twelve selected nitrogen lines are blended with molecular and atomic transitions. We are not sure of the oscillator strength of the blending lines and we do not have NLTE computations for these blending components. But for comparing the results from the four solar atlases this
Table 2. Selection of NI lines in the optical and near-IR bands

\begin{tabular}{lccc}
\hline$\lambda(\mathrm{nm})$ & $\mathrm{E}_{\text {low }}(\mathrm{eV})$ & $\log g f$ & $\mathrm{Q}$ \\
\hline 744.229 & 10.330 & -0.385 & $\mathrm{~B}+$ \\
746.831 & 10.336 & -0.190 & $\mathrm{~B}+$ \\
821.634 & 10.336 & +0.132 & $\mathrm{~B}+$ \\
822.314 & 10.330 & -0.271 & $\mathrm{~B}+$ \\
868.340 & 10.330 & +0.087 & $\mathrm{~B}+$ \\
871.883 & 10.336 & -0.336 & $\mathrm{~B}+$ \\
1010.513 & 11.750 & +0.219 & $\mathrm{~B}+$ \\
1011.248 & 11.758 & +0.607 & $\mathrm{~B}+$ \\
1011.464 & 11.764 & +0.768 & $\mathrm{~B}+$ \\
1050.700 & 11.840 & +0.094 & $\mathrm{~B}$ \\
1052.058 & 11.840 & +0.010 & $\mathrm{~B}$ \\
1053.957 & 11.844 & +0.503 & $\mathrm{~B}$ \\
\hline
\end{tabular}

Notes: Error of $\log g f \leq 0.03 \operatorname{dex}(Q=B+), \leq 0.08 \operatorname{dex}(Q=B)$.

is not a problem. The four lines we chose are the cleanest, although also for these lines blends or close-by lines are evident (see Figure 3). For this reason, being this one only a comparative analysis, we performed this exercise only with 1D models, for which the complete line list with blends is available.

With the ATLAS, HM and $\langle 3 \mathrm{D}\rangle$ solar models as input to SYNTHE, changing the nitrogen abundance, we 


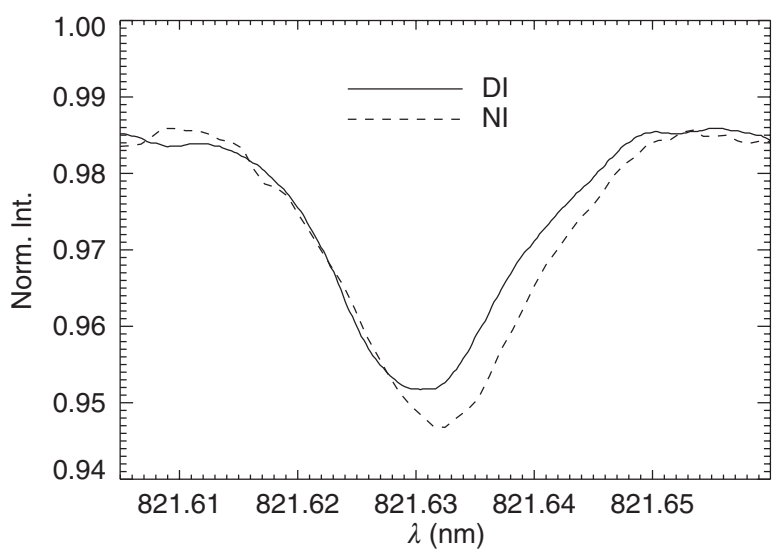

Figure 2 Observed profile of the Ni line at $\lambda 821.6 \mathrm{~nm}$ as extracted from the Delbouille (solid) and Neckel (dashed) solar disc-centre intensity spectral atlases. The differences between the two solar atlases are not easily explained; possibly telluric absorptions affect the Neckel profile.
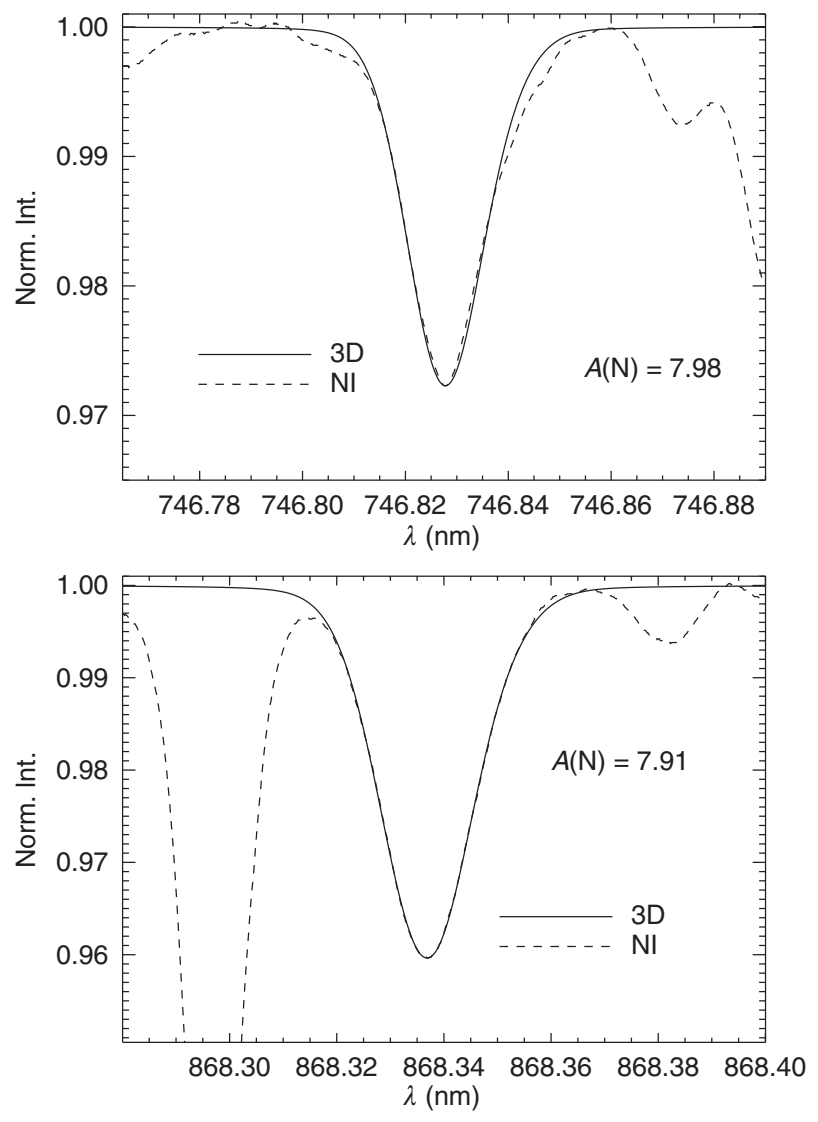

Figure 3 Observed profiles of two Ni lines are compared with a 3D synthetic profile.

computed three different grids of synthetic spectra. The fitting code, described in Caffau et al. (2005), is based on a $\chi^{2}$ minimisation, and for this purpose uses the MINUIT procedure.

Table 3 shows that the scatter in the abundance derived from the 821.6-nm line from the four solar atlases is considerably larger than what is obtained from the other two lines. The systematic uncertainty due to the choice of a
Table 3. $A(\mathrm{~N})$ of Ni from line fitting using three different 1D models

\begin{tabular}{lccccc}
\hline $\begin{array}{l}\text { Observed } \\
\text { spectrum }\end{array}$ & $\begin{array}{c}\lambda \\
(\mathrm{nm})\end{array}$ & $\mathrm{HM}$ & $\begin{array}{c}A(\mathrm{~N})_{\text {LTE }} \\
\text { ATLAS9 }\end{array}$ & $\langle 3 \mathrm{D}\rangle$ & $\begin{array}{c}A(\mathrm{~N})_{\text {LTE }} \\
\text { average }\end{array}$ \\
\hline $\mathrm{KF}$ & 746.8 & 7.861 & 7.824 & 7.809 & 7.873 \\
$\mathrm{NF}$ & & 7.874 & 7.837 & 7.827 & 7.899 \\
$\mathrm{NI}$ & & 7.890 & 7.834 & 7.823 & 7.922 \\
DI & & 7.908 & 7.849 & 7.840 & 7.883 \\
Average & & 7.883 & 7.836 & 7.825 & \\
Scatter & & 0.020 & 0.010 & 0.013 & $\mathrm{HM}$ \\
KF & 821.6 & 7.847 & 7.811 & 7.805 & 7.841 \\
NF & & 7.918 & 7.870 & 7.867 & 7.861 \\
NI & & 7.944 & 7.879 & 7.873 & 7.864 \\
DI & & 7.808 & 7.752 & 7.746 & 7.829 \\
Average & & 7.879 & 7.828 & 7.823 & \\
Scatter & & 0.063 & 0.059 & 0.060 & ATLAS9 \\
KF & 868.3 & 7.910 & 7.889 & 7.857 & 7.824 \\
NF & & 7.906 & 7.877 & 7.857 & 7.850 \\
NI & & 7.933 & 7.878 & 7.875 & 7.857 \\
DI & & 7.932 & 7.886 & 7.882 & 7.823 \\
Average & & 7.920 & 7.883 & 7.868 & \\
Scatter & & 0.014 & 0.006 & 0.013 & $\langle 3 \mathrm{D}\rangle$ \\
\hline
\end{tabular}

Notes. Column (1) is the observed spectrum identification: KF, Kurucz Flux; NF, Neckel Flux; NI, Neckel Intensity; and DI, Delbouille Intensity. Column (2) is the wavelength. Columns (3)-(5) are $A(\mathrm{~N})_{\text {LTE }}$ from line-profile fitting with HM, ATLAS9 and $\langle 3 \mathrm{D}\rangle$ models, respectively. Column (6) gives average values of the three lines for each atlas, using the model indicated in the last line of each block.

specific solar atlas that affects the abundance measurement is computed by comparing the average abundance obtained considering each atlas. This uncertainty is on average \pm 0.02 dex. We did not use $3 \mathrm{D}$ synthetic spectra for the fitting procedure. In fact, some tens of lines would be necessary to consider the whole range. 3D run are very time consuming and are, for the time being, not able to handle too many lines. Nevertheless, we compared the 3D synthetic profile to the observed solar spectra. Two examples are visible in Figure 3. Only nitrogen is considered in the $3 \mathrm{D}$ profile; for this reason the synthetic profile is not able to reproduce the complete shape of the feature (see line at $746.8 \mathrm{~nm}$ ) or gives a too high abundance (see line at $868.3 \mathrm{~nm}$ ).

\section{NLTE Computations}

The emergent flux depends on the LTE assumption. This is a good approximation for lines forming deeply in the photosphere, where the density is high. In fact LTE is valid if the photon mean free path is shorter than the distance over which matter temperature varies significantly. The photon mean free path depends on the probability for the photon to be thermalized, hence on the rate of the collisions between the absorbing atoms and electrons or hydrogen. This rate increases with matter density.

Since we do not yet have a code able to solve the NLTE problem for nitrogen in the case of a 3D model, we computed the departures from LTE for the $\langle 3 \mathrm{D}\rangle$ and the HM model using the Kiel code (Steenbock \& Holweger 


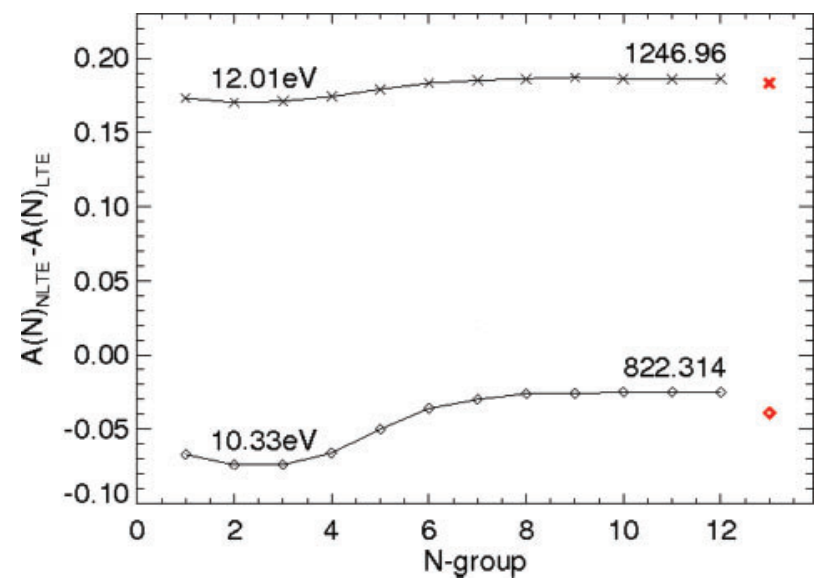

Figure 4 For two representative lines the NLTE corrections from the twelve group-averaged models, ordered according to increasing continuum intensity from left to right, are shown together with the result for the global $\langle 3 \mathrm{D}\rangle$ model (rightmost, bold symbol). The lowest curve is plotted at the true ordinate level, while the other is shifted up by 0.20 dex for clarity.

1984), with the model atom of Rentzsch-Holm (1996). To take into account excitation and ionisation of the nitrogen atoms by inelastic collisions with neutral hydrogen atoms, the Kiel code uses a generalisation of the formalism found in Drawin (1969). This formalism introduces a scaling factor, $S_{\mathrm{H}}$, that permits to modify the efficiency of collisions with hydrogen atoms $\left(0<S_{\mathrm{H}}<1\right)$. Currently we do not know which value of the scaling factor is the correct one and, therefore, decided to compute the NLTE corrections for the two extreme cases $\left(S_{\mathrm{H}}=0\right.$ and $\left.S_{\mathrm{H}}=1\right)$, and an intermediate value largely used in the Kiel community, $S_{\mathrm{H}}=\frac{1}{3}$. It could well be that each nitrogen transition we considered, actually require a different value of $S_{\mathrm{H}}$. Since, in any case, the NLTE corrections are small, we shall consider this differential effect as negligible.

We considered the effects of the horizontal temperature fluctuations on the NLTE correction. The procedure we used is similar to the one that Aufdenberg, Ludwig \& Kervella (2005) used to estimate the effects of horizontal temperature inhomogeneities. We ordered the emerging flux as a function of temperature. We divided into 12 bins in increasing temperature and produced horizontally and time-averaged models. We computed the NLTE corrections for these twelve average models.

From the results of this 1D-NLTE computation for each line we found that the NLTE corrections are small and not exceeding -0.05 dex. Regarding the effects of the horizontal temperature inhomogeneities, we found that lines with lower excitation energy are more sensitive than lines with higher excitation energy that are formed deep in the photosphere. But these effects are small for our sample of nitrogen lines, since all of them have high excitation potential (cf. Table 2). From Figure 4 we can see that the horizontal variation of the NLTE corrections is $0.05 \mathrm{dex}$ at most. For this reason we expect that a full 3D-NLTE computation would not differ from our 1D-NLTE calculation by more than 0.03 dex.

\section{Nitrogen Abundance}

Our final 3D-NLTE nitrogen abundance is obtained by averaging the individual 3D-NLTE abundances of each line with equal weight. These abundances are obtained with the spectrum synthesis code LINFOR $3 \mathrm{D}$ (see Table 4). In the same table we show the total 3D corrections, defined as $A(\mathrm{~N})_{3 \mathrm{D}^{-}}-A(\mathrm{~N})_{1 \mathrm{D}_{\mathrm{LHD}}}$, and the granulation correction, defined as $A(\mathrm{~N})_{3 \mathrm{D}}-A(\mathrm{~N})_{\langle 3 \mathrm{D}\rangle}$, for each line. The latter corrections, Column (12), are negative for all lines, indicating that the horizontal temperature fluctuations systematically strengthen the (high-excitation) lines. The total 3D corrections, Column (13) are systematically more positive, because the $1 \mathrm{D}_{\text {LHD }}$ model produces slightly stronger lines than the $\langle 3 \mathrm{D}\rangle$ model. The $3 \mathrm{D}$ corrections are of the same order of magnitude as the NLTE corrections.

Our final result for the solar photospheric nitrogen abundance is:

$$
\begin{array}{ll}
A(\mathrm{~N})=7.85 \pm 0.12 & \text { for } S_{\mathrm{H}}=0 \\
A(\mathrm{~N})=7.86 \pm 0.12 & \text { for } S_{\mathrm{H}}=\frac{1}{3} \\
A(\mathrm{~N})=7.87 \pm 0.12 & \text { for } S_{\mathrm{H}}=1
\end{array}
$$

If only the EWs from Biémont et al. (1990) are considered, $A(\mathrm{~N})$ is the same while the scatter is reduced to 0.06 dex.

\section{Discussion}

In theory, the same nitrogen abundance should be derived from each line. In practice, this is not the case due to uncertainties in the analysis, related to the following elements: the model atmosphere, the values of $\log g f$, and the EWs. The results discussed in the following refer to the $3 \mathrm{D}_{\mathrm{NLTE}}$ model, assuming $S_{\mathrm{H}}=\frac{1}{3}$.

Concerning the $\log g f$ values and referring to Table 2, one could select only lines with the $\mathrm{Q}$ value equal to $\mathrm{B}+$ and consider the abundances derived only from these lines. From the results of Table 4 we find that selecting only lines with $Q=B+$ does not reduce the scatter (both for results from the $3 \mathrm{D}$ and the $\mathrm{HM}$ model) and the mean value becomes $A(\mathrm{~N})=7.83$ dex, very close to our recommended value, $A(\mathrm{~N})=7.86$ dex.

The EWs of Biémont et al. (1990) are slightly different from that of Grevesse et al. (1990) by 1 to 6 percent at most. This is an indication that these EWs are reliable. A possible way to try to decrease the scatter is to select the lines for which we have the EWs both from Grevesse et al. (1990) and Biémont et al. (1990). Since both the two authors chose these lines, we can consider this subset more reliable. We find that the mean value does not change, while the scatter for the nitrogen abundance from Grevesse et al. (1990) decreases to 0.07 dex. A different possibility is to take the four lines, namely the lines $821.6 \mathrm{~nm}, 871.8 \mathrm{~nm}$, $1011.2 \mathrm{~nm}$ and $1011.4 \mathrm{~nm}$ for which EWs of the previous authors differ by less than three percent. This agreement may be taken to imply that the EWs of these lines are more reliable than the others. The results from this subset are $A(\mathrm{~N})=7.88 \pm 0.06$ with EWs of Grevesse et al. 
Table 4. $A(\mathrm{~N})_{\mathrm{LTE}}, A(\mathrm{~N})_{\mathrm{NLTE}}$ and 3D corrections for selected NI lines

\begin{tabular}{|c|c|c|c|c|c|c|c|c|c|c|c|c|}
\hline \multirow{2}{*}{$\begin{array}{l}\lambda \\
(\mathrm{nm}) \\
(1)\end{array}$} & \multicolumn{2}{|c|}{$\begin{array}{l}\text { EW } \\
(\mathrm{pm})\end{array}$} & \multicolumn{2}{|c|}{$A(\mathrm{~N})_{\text {LTE }} 3 \mathrm{D}$} & \multicolumn{2}{|c|}{$\begin{array}{c}A(\mathrm{~N})_{\text {NLTE }} 3 \mathrm{D} \\
S_{\mathrm{H}}=\frac{1}{3}\end{array}$} & \multicolumn{2}{|c|}{$\begin{array}{c}A(\mathrm{~N})_{\text {NLTE }} 3 \mathrm{D} \\
S_{\mathrm{H}}=1\end{array}$} & \multicolumn{2}{|c|}{$\begin{array}{c}A(\mathrm{~N})_{\mathrm{NLTE}} 3 \mathrm{D} \\
S_{\mathrm{H}}=0\end{array}$} & \multirow{2}{*}{$\begin{array}{c}\text { 3D- }\langle 3 \mathrm{D}\rangle \\
(\mathrm{dex}) \\
(12)\end{array}$} & \multirow{2}{*}{$\begin{array}{c}3 \mathrm{D}-1 \mathrm{D}_{\mathrm{LHD}} \\
\left(\alpha_{\mathrm{MLT}}=1.0\right) \\
(\mathrm{dex}) \\
(13)\end{array}$} \\
\hline & $\begin{array}{l}\mathrm{G} \\
(2)\end{array}$ & $\begin{array}{l}\text { B } \\
(3)\end{array}$ & $\begin{array}{l}G \\
(4)\end{array}$ & $\begin{array}{l}\text { B } \\
(5)\end{array}$ & $\begin{array}{l}G \\
(6)\end{array}$ & $\begin{array}{l}\text { B } \\
(7)\end{array}$ & $\begin{array}{l}\mathrm{G} \\
(8)\end{array}$ & $\begin{array}{l}\text { B } \\
(9)\end{array}$ & $\begin{array}{c}\mathrm{G} \\
(10)\end{array}$ & $\begin{array}{l}\mathrm{B} \\
(11)\end{array}$ & & \\
\hline 744.2 & 0.26 & 0.27 & 7.808 & 7.826 & 7.774 & 7.792 & 7.782 & 7.800 & 7.770 & 7.788 & -0.039 & -0.002 \\
\hline 746.8 & 0.52 & 0.49 & 7.961 & 7.931 & 7.923 & 7.893 & 7.931 & 7.901 & 7.919 & 7.889 & -0.033 & +0.008 \\
\hline 821.6 & 0.86 & 0.87 & 7.854 & 7.860 & 7.802 & 7.808 & 7.817 & 7.823 & 7.790 & 7.796 & -0.039 & +0.007 \\
\hline 822.3 & 0.24 & & 7.593 & & 7.554 & & 7.565 & & 7.544 & & -0.055 & -0.018 \\
\hline 868.3 & 0.78 & 0.81 & 7.828 & 7.849 & 7.781 & 7.802 & 7.794 & 7.815 & 7.767 & 7.788 & -0.037 & +0.007 \\
\hline 871.8 & 0.42 & 0.43 & 7.927 & 7.939 & 7.887 & 7.899 & 7.898 & 7.910 & 7.875 & 7.887 & -0.044 & -0.006 \\
\hline 1010.5 & 0.18 & & 7.956 & & 7.939 & & 7.944 & & 7.931 & & -0.066 & -0.020 \\
\hline 1011.2 & 0.35 & 0.36 & 7.897 & 7.912 & 7.878 & 7.893 & 7.883 & 7.898 & 7.869 & 7.884 & -0.060 & -0.011 \\
\hline 1011.4 & 0.55 & 0.54 & 7.976 & 7.966 & 7.955 & 7.945 & 7.961 & 7.951 & 7.937 & 7.927 & -0.053 & -0.001 \\
\hline 1050.7 & 0.14 & & 8.002 & & 7.992 & & 7.995 & & 7.986 & & -0.064 & -0.020 \\
\hline 1052.0 & 0.08 & & 7.829 & & 7.819 & & 7.822 & & 7.812 & & -0.067 & -0.024 \\
\hline 1053.9 & 0.32 & & 7.989 & & 7.978 & & 7.980 & & 7.971 & & -0.057 & -0.010 \\
\hline Average & & & 7.885 & 7.890 & 7.857 & 7.862 & 7.864 & 7.871 & 7.848 & 7.851 & -0.051 & -0.008 \\
\hline Scatter & & & 0.114 & 0.053 & 0.122 & 0.060 & 0.120 & 0.058 & 0.122 & 0.059 & & \\
\hline
\end{tabular}

Notes. EWs from the literature using LINFOR3D. Columns labelled 'G' are from Grevesse et al. (1990), labelled 'B' are from Biémont et al. (1990). Column (1) is the wavelength, columns (2)-(3) the EWs, columns (4)-(5) $A(\mathrm{~N})_{\text {LTE }}$ from 3D model, columns (6)-(11) A(N) NLTE from 3D model for various values of $S_{\mathrm{H}}$, columns (12)-(13) two different 3D corrections.

(1990) and $A(\mathrm{~N})=7.89 \pm 0.06$ with EWs of Biémont et al. (1990). From this subset, it can be reasonable to discard the line at $1011.4 \mathrm{~nm}$. In fact we could not obtain a good fit for this line and the correspondent abundance was too high if compared with the fitting results of the other three lines (see Section 3). This behaviour is the same for each model and each atlas we used. The nitrogen abundance we obtain from these three lines is $A(\mathrm{~N})=7.86 \pm 0.05$ dex with EWs of Grevesse et al. (1990) and $A(\mathrm{~N})=7.87 \pm 0.05$ dex with EWs of Biémont et al. (1990). With this selection the scatter is further decreased for both sets of EWs.

We conclude from this exercise that the mean nitrogen abundances given above are robust against exclusion of the lines which have either the more uncertain $\log g f$ values or exclusion of the lines for which we consider the EWs to be less reliable.

Our preferred value for the solar nitrogen abundance is $A(\mathrm{~N})=7.86 \pm 0.12$, assuming the NLTE correction with $S_{\mathrm{H}}=\frac{1}{3}$. The indicated error represents the line-toline scatter and does not include any uncertainty in the $\log g f$ values.

\section{References}

Allende Prieto, C., Lambert, D. L. \& Asplund, M., 2001, ApJ, 556, L63

Asplund, M., Grevesse, N., Sauval, A. J., Allende Prieto, C. \& Kiselman, D., 2004, A\&A, 417, 751

Asplund, M., Grevesse, N. \& Sauval, A. J., 2005, ASPC, 336, 25

Aufdenberg, J. P., Ludwig, H.-G. \& Kervella, P., 2005, ApJ, 633, 424

Basu, S. \& Antia, H. M., 2008, PhR, 457, 217

Biémont, E., Froese Fischer, C., Godefroid, M., Vaeck, N. \& Hibbert, A., 1990, in Proc. 3rd International Colloquium of the Royal Netherlands Academy of Arts and Sciences, Ed. Hansen, J. E. (Amsterdam: North-Holland), 59
Caffau, E. \& Ludwig, H.-G., 2007, A\&A, 467, L11

Caffau, E., Bonifacio, P., Faraggiana, R., François, P., Gratton, R. G. \& Barbieri, M., 2005, A\&A, 441, 533

Caffau, E., Ludwig, H.-G., Steffen, M., Ayres, T. R., Bonifacio, P., Cayrel, R., Freytag, B. \& Plez, B., 2008, A\&A, 488, 1031

Castelli, F., 2005, MmSAI, 8, 44

Castelli, F. \& Kurucz, R. L., 2003, IAUS, 210, A20

Delbouille, L., Roland, G. \& Neven, L., 1973, Atlas photometrique du spectre solaire de $\lambda 3000$ a $\lambda 10000$ (Liege: Universite de Liege, Institut d'Astrophysique)

Delbouille, L., Roland, G., Brault, J. \& Testerman, L., 1981, Photometric Atlas of the Solar Spectrum from 1850 to $10000 \mathrm{~cm}^{-1}$, http: //bass2000.obspm.fr/solar_spect.php

Drawin, H. W., 1969, ZPhy, 225, 483

Freytag, B., Steffen, M. \& Dorch, B., 2002, AN, 323, 213

Freytag, B., Steffen, M., Wedemeyer-Böhm, S. \& Ludwig, H.-G., 2003, CO5BOLD User Manual, http: / / www . astro.

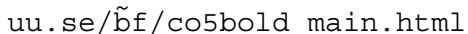

Grevesse, N., Lambert, D. L., Sauval, A. J., van Dishoeck, E. F., Farmer, C. B. \& Norton, R. H., 1990, A\&A, 232, 225

Grevesse, N. \& Sauval, A. J., 1998, SSRv, 85, 161

Holweger, H., 1967, ZA, 65, 365

Holweger, H. \& Müller, E. A., 1974, SoPh, 39, 19

Kurucz, R., 1993a, ATLAS9 Stellar Atmosphere Programs and $2 \mathrm{~km} / \mathrm{s}$ grid (Cambridge, Mass.: Smithsonian Astrophysical Observatory)

Kurucz, R., 1993b, SYNTHE Spectrum Synthesis Programs and Line Data (Cambridge, Mass.: Smithsonian Astrophysical Observatory)

Kurucz, R. L., 2005a, MSAIS, 8, 189

Kurucz, R. L., 2005b, MSAIS, 8, 14

Neckel, H. \& Labs, D., 1984, SoPh, 90, 205

Rentzsch-Holm, I., 1996, A\&A, 305, 275

Sbordone, L., 2005, MSAIS, 8, 61

Sbordone, L., Bonifacio, P., Castelli, F. \& Kurucz, R. L., 2004, MSAIS, 5, 93

Steenbock, W. \& Holweger, H., 1984, A\&A, 130, 319

Wedemeyer, S., Freytag, B., Steffen, M., Ludwig, H.-G. \& Holweger, H., 2004, A\&A, 414, 1121 